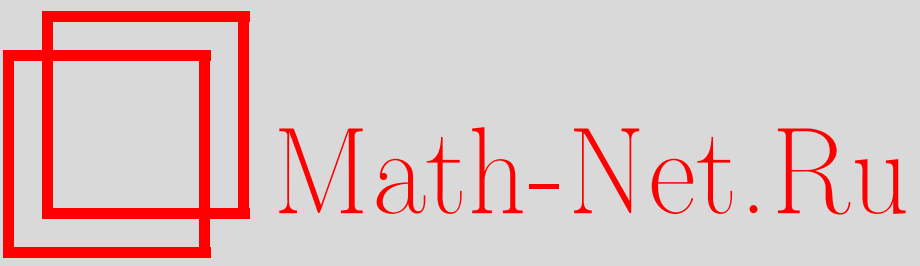

С. Я. Старцев, Об инвариантах Лапласа гиперболических уравнений, линеаризуемых дифференциальной подстановкой, ТМФ, 1999, том 120, номер 2, 237-247

DOI: https://doi.org/10.4213/tmf772

Использование Общероссийского математического портала Math-Net.Ru подразумевает, что вы прочитали и согласны с пользовательским соглашением

http://www . mathnet.ru/rus/agreement

Параметры загрузки:

IP : 3.81 .55 .215

26 апреля 2023 г., 16:11:45 


\title{
ФИЗИКА
}

Том 120, № 2

август, 1999

\author{
(C) 1999 г. \\ С.Я. Старцев*
}

\section{ОБ ИНВАРИАНТАХ ЛАПЛАСА ГИПЕРБОЛИЧЕСКИХ УРАВНЕНИЙ, ЛИНЕАРИЗУЕМЫХ ДИФФЕРЕНЦИАЛЬНОЙ ПОДСТАНОВКОЙ}

Показано, что необходимым условием наличия у скалярного гиперболического уравнения дифференциальной подстановки, переводящей решения этого уравнения в решения линейного гиперболического уравнения, является ограниченность порядков обобщенных инвариантов Лапласа этого уравнения.

\section{1. ВВЕДЕНИЕ}

В ряде работ последних лет [1-8] показано, что многие важные свойства уравнения вида

$$
u_{x y}=F\left(x, y, u, u_{x}, u_{y}\right)
$$

могут быть описаны в терминах обобщенных инвариантов Лапласа $H_{i}$ этого уравнения, задаваемых формулами

$$
\begin{gathered}
H_{-1}=F_{u}+F_{u_{x}} F_{u_{y}}-D_{x}\left(F_{u_{x}}\right), \quad H_{0}=F_{u}+F_{u_{x}} F_{u_{y}}-D_{y}\left(F_{u_{y}}\right), \\
2 H_{i}-H_{i+1}-H_{i-1}=D_{x} D_{y}\left(\ln H_{i}\right)
\end{gathered}
$$

$D_{x}$ и $D_{y}$ - операторы полных производных по $x$ и $y$ в силу уравнения (1). Смешанные частные производные $\partial^{i+j} u / \partial x^{i} \partial y^{j}, i, j \neq 0$, мы исключаем в силу уравнения (1) и его дифференциальных следствий. В оставшихся переменных $x, y, u, u_{i}=\partial^{i} u / \partial x^{i}$, $v_{i}=\partial^{i} u / \partial y^{i}$ полные производные $D_{x}$ и $D_{y}$ выражаются формулами

$$
\begin{aligned}
& D_{x}=\frac{\partial}{\partial x}+u_{1} \frac{\partial}{\partial u}+\sum_{i=1}^{\infty}\left(u_{i+1} \frac{\partial}{\partial u_{i}}+D_{y}^{i-1}(F) \frac{\partial}{\partial v_{i}}\right) \\
& D_{y}=\frac{\partial}{\partial y}+v_{1} \frac{\partial}{\partial u}+\sum_{i=1}^{\infty}\left(v_{i+1} \frac{\partial}{\partial v_{i}}+D_{x}^{i-1}(F) \frac{\partial}{\partial u_{i}}\right) .
\end{aligned}
$$

В работах [1-4] было доказано, что обрашение в нуль при некоторых $i \geqslant 0$ и $j<0$ членов последовательности инвариантов Лапласа $H_{i}$ является критерием интегрируемости уравнения (1) по Дарбу. Обрыв цепочки инвариантов Лапласа (т.е. обращение

${ }^{*}$ Институт математики с Вычислительным центром Уфимского научного центра, Уфа, Россия 
одного из инвариантов в нуль) является также необходимым и достаточным условием наличия у уравнения (1) симметрий, зависяших от произвольной функции (см. [2, 5, 7]).

Однако условия на инварианты Лапласа, выделяюшие замечательные в том или ином смысле уравнения (1), не исчерпываются обрывом цепочки инвариантов Лапласа. Для того чтобы понять характер этих условий, нам потребуется ввести некоторые обозначения. Заметим сначала, что то, что мы будем называть здесь функциями, может зависеть не только от $x$ и $y$, но и, вообше говоря, от $u$ и ее частных производных $u_{i}$ и $v_{i}$. Будем говорить, что функция $f$ имеет порядок $(k, m)$, если она не зависит от переменных $v_{i}$, $i>k, u_{j}, j>m$, и $f_{v_{k}} \neq 0, f_{u_{m}} \neq 0$. Если функция $f$ имеет порядок $(k, m)$, мы будем писать $\operatorname{ord}_{y}(f)=k, \operatorname{ord}_{x}(f)=m$, а также $\operatorname{ord}(f)=(k, m)$.

Нетрудно видеть, что в ситуации обшего положения порядок инвариантов Лапласа $H_{i}$ растет с ростом абсолютного значения индекса $i$. Например, $\operatorname{ord}_{x}\left(H_{i}\right)$ при $i \geqslant 0$, вообще говоря, равен $i+1$. Между тем для "хороших" уравнений порядок инвариантов Лапласа может оказаться меньше указанного выше максимума.

Впервые этот факт был замечен, по-видимому, в работе [8], где было показано, что из выполнения важного в ряде ситуаций условия

$$
F_{u_{x}}=D_{y}(a), \quad \operatorname{ord}_{x}(a)=k
$$

следует, что $\left(H_{i}\right)_{u_{i+1}}=0$ при некотором неотрицательном $i \leqslant k$. Условие $(2)$ выполняется, например, когда решения уравнения (1) подстановкой $k$-го порядка переводятся в решения линейного уравнения

$$
w_{x y}+\alpha(x, y) w_{x}+\beta(x, y) w_{y}+\gamma(x, y) w=0,
$$

а также в случае существования для уравнения (1) подстановки, переводящей его в какое-нибудь из уравнений Клейна-Гордона $w_{x y}=F(x, y, w)$. Примером уравнения последнего типа является уравнение $u_{x y}=\sqrt{\left(1-4 u_{x}^{2}\right) u_{y}}$, которое переводится подстановкой $w=\arcsin \sqrt{1-4 u_{x}^{2}}$ в уравнение $w_{x y}=\mp \sin w$. Непосредственным вычислением нетрудно убедиться, что для этого уравнения $\left(H_{1}\right)_{u_{2}}=0$.

Достаточно правдоподобной также представляется гипотеза о том, что инварианты Лапласа уравнения (1), получаюшегося дифференциальной подстановкой из линейного уравнения вида (3), имеют нулевой порядок по одной из характеристик, т.е. не зависят либо от $u_{i}$, либо от $v_{i}$ (заметим, что эта гипотеза почти доказана в разделе 4 , см. утверждение 2$)$. В качестве примера рассмотрим уравнение $u_{x y}=A(x, y) e^{u} u_{x}+$ $A_{x}(x, y) e^{u}-e^{-u} u_{y}+C(x, y)$, которое связано подстановкой $u=-\ln \left(w_{x} / w+\beta\right)$ с линейным уравнением (3), если $A=\gamma-\alpha \beta-\beta_{y}$ и $C=\alpha_{x}-\beta_{y}$ (см. [9]). Инварианты Лапласа $H_{i}$ этого уравнения не зависят от $u_{j}$ при любых $i<0$ и $j>0$.

Объектом изучения в настояшей работе является ситуация, "обратная" описанной в предыдущем абзаце. Заметим, что интегрируемость уравнения (1) по Дарбу означает, что найдутся дифференциальные подстановки, переводящие уравнение (1) в уравнение $w_{x}=0$ или в уравнение $\bar{w}_{y}=0$. Поэтому обобщением понятия интегрируемости по Дарбу естественно считать сушествование для уравнения (1) дифференциальной подстановки, переводящей решения этого уравнения в решения линейного уравнения вида (3). В качестве примера уравнения, сводимого к (3), можно привести известное 
еше Гурса [10] уравнение $u_{x y}=\sqrt{u_{x} u_{y}}$, которое переводится подстановкой $w=\sqrt{u_{x}}$ в уравнение $w_{x y}=(1 / 4) w$ и при этом не является интегрируемым по Дарбу. Естественно ожидать, что упомянутый выше критерий интегрируемости по Дарбу хотя бы частично (необходимость) может быть перенесен на эту более общую ситуацию.

Основным результатом настояшей работы является доказательство того, что из существования для уравнения (1) подстановки $w=g, \operatorname{ord}_{x}(g)=k>0$, переводящей решения уравнения (1) в решения уравнения (3), следует ограниченность порядков инвариантов Лапласа уравнения (1), а именно

$$
\operatorname{ord}_{x}\left(H_{i}\right) \leqslant k \text { при } i \geqslant 0 \text {. }
$$

Заметим, что $\operatorname{ord}_{y}\left(H_{i}\right)$ при этом не обязательно ограничен и может расти с максимальной скоростью, - забвение этого факта часто приводит к путанице. Оценка (4) верна, очевидно, и в случае интегрируемости уравнения (1) по Дарбу - просто обрыв цепочки инвариантов в этом случае произойдет до того, как порядок инвариантов сможет превзойти $k$.

Конструктивность полученных условий линеаризуемости примерно та же, что и для упомянутых выше условий интегрируемости по Дарбу: их проверка для конкретного уравнения сводится к простому вычислению. Однако заранее неизвестно, до какого значения $i$ нужно считать инварианты Лапласа. Посчитав несколько первых инвариантов и увидев, что их порядок достигает величины $m$, мы лишш можем гарантировать, что для уравнения не сушествует линеаризуюших его подстановок порядка, меньшего $m+1$.

Ограниченность порядков инвариантов Лапласа является сушественно более жестким условием по сравнению с полученным в работе [8] условием линеаризуемости $\left(\left(H_{i}\right)_{u_{i+1}}=0\right.$ при некотором неотрицательном $\left.i \leqslant k\right)$. Но достаточным это условие все же не является. Действительно, нетрудно проверить, что инварианты Лапласа уравнения

$$
u_{x y}=f\left(y, u, u_{y}\right) u_{x}
$$

не зависят от $u_{i}$ для всех $i>1$. Между тем здравый смысл подсказывает, что это уравнение лишь для некоторых $f$ может быть линеаризовано подстановкой $w=g, \operatorname{ord}_{x}(g)>0$. По крайней мере, можно строго доказать, что, например, для уравнения $u_{x y}=e^{u_{y}} u_{x}$ не существует линеаризующих его подстановок, зависящих от $u_{i}$. Некоторый оптимизм, однако, внушает тот факт, что уравнение (5) линеаризуется не зависящей от $u_{i}$ подстановкой $w=P\left(y, u, u_{y}\right)$, где $P$ находится из условия $P_{u_{y}} f+P_{u}=0$, которая переводит это уравнение в $w_{x}=0$.

\section{2. ПРЕДВАРИТЕЛЬНЫЕ СВЕДЕНИЯ}

В настоящей статье мы постоянно будем пользоваться обобщенными преобразованиями и инвариантами Лапласа. Но перед тем как перейти к их формальному определению, дадим описание преобразования Лапласа.

Рассмотрим дифференциальный оператор вида

$$
Z=D_{x} D_{y}+a D_{x}+b D_{y}+c
$$


где $a, b$ и $c$ - некоторые функции. Этот оператор можно записать как в виде

$$
Z=\left(D_{y}+a\right) \circ\left(D_{x}+b\right)-H_{0}, \quad H_{0}=D_{y}(b)+a b-c
$$

так и в виде

$$
Z=\left(D_{x}+b\right) \circ\left(D_{y}+a\right)-K_{0}, \quad K_{0}=D_{x}(a)+a b-c .
$$

Пусть $K_{0} \neq 0$. Тогда, как нетрудно проверить, выполнено соотношение

$$
\begin{aligned}
& \left(D_{y}+a_{1}\right) \circ\left(\left(D_{x}+b\right) \circ\left(D_{y}+a\right)-K_{0}\right)= \\
& \quad=\left(\left(D_{y}+a_{1}\right) \circ\left(D_{x}+b\right)-K_{0}\right) \circ\left(D_{y}+a\right)
\end{aligned}
$$

где $a_{1}=a-D_{y}\left(\ln K_{0}\right)$. Формула $(7)$ означает, что оператор $\left(D_{y}+a\right)$ переводит ядро $Z$ в ядро оператора

$$
Z_{-1}=\left(D_{y}+a_{1}\right) \circ\left(D_{x}+b\right)-K_{0}
$$

Оператор $Z_{-1}$ будем называть $y$-преобразованием Лапласа оператора $Z$.

В свою очередь мы можем переписать $Z_{-1}$ в виде

$$
Z_{-1}=\left(D_{x}+b\right) \circ\left(D_{y}+a_{1}\right)-K_{1}
$$

где, как нетрудно убедиться,

$$
K_{1}=2 K_{0}-D_{x} D_{y}\left(\ln K_{0}\right)-H_{0}
$$

и построить $y$-преобразование Лапласа оператора $Z_{-1}$, которое мы обозначим через $Z_{-2}$, и т.д. Многократное применение описанной вьше процедуры дает нам последовательность операторов

$$
Z_{-i}=\left(D_{x}+b\right) \circ\left(D_{y}+a_{i}\right)-K_{i}=\left(D_{y}+a_{i}\right) \circ\left(D_{x}+b\right)-K_{i-1},
$$

где $K_{i}, a_{i}$ определяются рекуррентными формулами

$$
\begin{aligned}
K_{i+1} & =2 K_{i}-D_{x} D_{y}\left(\ln K_{i}\right)-K_{i-1}, \quad H_{-1}=K_{0} \\
a_{i+1} & =a_{i}-D_{y}\left(\ln K_{i}\right), \quad a_{0}=a .
\end{aligned}
$$

Аналогичным образом описывается и $x$-преобразование Лапласа - надо лиш везде (исключая, конечно, формулу (6)) поменять местами $x$ и $y$, а также $a$ и $b$.

Подытожим вышесказанное в следуюшем определении. 
ОПРЕДЕЛЕНИЕ 1. Функции

$$
H_{0}=D_{y}(b)+a b-c, \quad K_{0}=D_{x}(a)+a b-c
$$

называются соответственно главными $x$ - и $y$-инвариантами Лапласа оператора (6).

Последуюшие $x$-инварианты Лапласа $H_{i}$ определяются рекуррентной формулой

$$
H_{i+1}=2 H_{i}-D_{x} D_{y}\left(\ln H_{i}\right)-H_{i-1},
$$

где $H_{-1}=K_{0}$, а последующие $y$-инварианты Лапласа $K_{i}-$ формулой

$$
K_{i+1}=2 K_{i}-D_{x} D_{y}\left(\ln K_{i}\right)-K_{i-1}
$$

где $K_{-1}=H_{0}$.

Операторы

$$
\begin{aligned}
& Z_{i}=\left(D_{y}+a\right) \circ\left(D_{x}+b_{i}\right)-H_{i}, \quad b_{i}=b_{i-1}-D_{x}\left(\ln H_{i-1}\right), \quad b_{0}=b, \\
& Z_{-i}=\left(D_{x}+b\right) \circ\left(D_{y}+a_{i}\right)-K_{i}, a_{i}=a_{i-1}-D_{y}\left(\ln K_{i-1}\right), a_{0}=a,
\end{aligned}
$$

называются соответственно $i$-ми $x$ - и $y$-преобразованиями Лапласа оператора (6).

Инвариантами Лапласа уравнения (1) называются инварианты Лапласа его оператора линеаризации

$$
L=D_{x} D_{y}-F_{u_{x}} D_{x}-F_{u_{y}} D_{y}-F_{u}
$$

Мы можем продолжать цепочку преобразований и инвариантов Лапласа лишь до тех пор, пока очередной инвариант Лапласа не обратится в нуль. В дальнейшем, не оговаривая это каждый раз, мы будем иметь в виду, что все сформулированные ниже утверждения об инвариантах Лапласа верны лишь для тех из них, которые мы можем построить, - для инвариантов, предшествуюших нулевому, если таковой найдется, а если ни один из инвариантов не обрашается в нуль, то просто для всех инвариантов Лапласа.

ОПРЕДЕЛЕнИЕ 2. Функцию $Q$ будем называть $x$-интегралом ( $y$-интегралом) уравнения $(1)$, если $D_{y}(Q)=0\left(D_{x}(Q)=0\right)$. Если $Q$ зависит только от $x$ (только от $\left.y\right)$, то $Q$ будем называть тривиальным $x$-интегралом ( $y$-интегралом).

Нетрудно видеть, что $x$-интеграл ( $y$-интеграл) не может зависеть от переменных $v_{i}$ $\left(u_{i}\right)$. Порядок старшей из частных производных, от которых интеграл сушественно зависит, называется порядком интеграла.

В работах $[1,2]$ было получено следуюшее

УТВЕРЖДЕнИЕ 1. Если уравнение (1) допускает нетривиальный $x$-интеграл (у-интеграл) порядка $k$, то $H_{i}=0\left(K_{i}=0\right)$ при некотором $i<k$, где через $H_{i}$ $\left(K_{i}\right)$ обозначень $x$-инварианты ( $($-инварианты) Лапласа уравнения (1).

3 Теоретическая и математическая физика, т. 120, № 2, 1999 г. 
ОПРЕДЕлЕниЕ 3 . Будем говорить, что уравнение (1) линеаризуется подстановкой $w=g, \operatorname{ord}(g)=(k, m), k^{2}+m^{2} \neq 0$, если подстановка переводит любое решение $(1)$ в решение уравнения вида (3). На более формальном языке это означает, что найдутся функции $\alpha, \beta$ и $\gamma$, зависящие только от $x$ и $y$, такие что

$$
\left(D_{x} D_{y}+\alpha(x, y) D_{x}+\beta(x, y) D_{y}+\gamma(x, y)\right)(g)=0 .
$$

Заметим, что уравнения с нетривиальными интегралами удовлетворяют определению 3 , так как интеграл задает подстановку, переводяшую решения уравнения (1) в решения уравнения $w_{x y}=0$. Таким образом, наличие у уравнения $(1)$ нетривиального интеграла является частным случаем ситуации, описанной в определении 3, и естественно ожидать, что утверждение 1 может быть обобщено на случай уравнений, линеаризующихся дифференциальной подстановкой. Результат настоящей работы, строгая формулировка которого приведена в разделе 3 , можно рассматривать как прямое обобшение этого утверждения.

\section{3. ОСНОВНАЯ ТЕОРЕМА И ДОКАЗАТЕЛЬСТВО ВСПОМОГАТЕЛЬНЫХ УТВЕРЖДЕНИЙ}

ТеОрема. Пусть уравнение (1) линеаризуется подстановкой $w=g, \operatorname{ord}_{x}(g)=$ $k>0\left(\operatorname{ord}_{y}(g)=k>0\right)$. Тогда его $x$-инварианты Лапласа $H_{i}\left(y\right.$-инварианты $\left.K_{i}\right)$ удовлетворяют условию $\operatorname{ord}_{x}\left(H_{i}\right) \leqslant k\left(\operatorname{ord}_{y}\left(K_{i}\right) \leqslant k\right)$.

Для уравнений с нетривиальными интегралами теорема выполняется специфическим образом - в силу утверждения 1 инварианты Лапласа обратятся в нуль до того, как их порядок сможет превзойти $k$.

Для доказательства теоремы необходимо решить три не равноценные по сложности задачи, сформулированные в виде вспомогательных утверждений, а именно:

1) показать, что порядки инвариантов Лапласа уравнения, линеаризуюшегося подстановкой, не могут бесконечно расти, а ограничены какой-нибудь величиной (не обязательно равной $k$ ), т.е. показать, что найдется $n$ такое, что $\operatorname{ord}_{x}\left(H_{i}\right) \leqslant n$;

2) доказать, что из линеаризуемости уравнения (1) подстановкой $w=g, \operatorname{ord}_{x}(g)=$ $k>0$, следует $F_{u_{x}}=D_{y}\left(\xi(x, y)-\ln \left(g_{u_{k}}\right)\right)$;

3 ) показать, что если $\operatorname{ord}_{x}\left(H_{i}\right)$ ограничен и выполнено соотношение $F_{u_{x}}=D_{x}(s)$, где $\operatorname{ord}_{x}(s)=m$, то $\operatorname{ord}_{x}\left(H_{i}\right) \leqslant \max \{m, 1\}$.

Ясно, что если удастся доказать эти три утверждения, то мы докажем и теорему. Точнее говоря, мы докажем тем самым несколько больше: $\operatorname{ord}_{x}\left(H_{i}\right) \leqslant \operatorname{ord}_{x}\left(g_{u_{k}}\right)$. Kроме того, если удастся показать, что порядок $g_{u_{k}}$ ограничен не $k$, а меньшей величиной, то это автоматически приведет к усилению нашей оценки порядков инвариантов Лапласа.

В этом разделе мы докажем 2-е и 3-е утверждения, а более сложному первому посвятим раздел 4.

Доказательство 2-го вспомогательного утверждения самое простое. Для его обоснования достаточно собрать в соотношении (13) члены при $u_{k+1}$. Учитывая формулу $D_{y}\left(u_{k+1}\right)=D_{x}^{k}(F)=F_{u_{x}} u_{k+1}+\cdots$, которую нетрудно проверить по индукции, получим равенство

$$
\left(D_{y}+F_{u_{x}}+\beta\right)\left(g_{u_{k}}\right)=0 \text {, }
$$


из которого и вытекает требуемое соотношение.

Доказательство 3-го вспомогательного утверждения проведем от противного. Пусть найдется $l$ такое, что $\operatorname{ord}_{x}\left(H_{l}\right)>\max \{m, 1\}$. Выберем $l$ так, чтобы оно было минимальным числом, удовлетворяющим этому условию. Очевидно, что $l>0$.

Поскольку порядок инвариантов Лапласа ограничен, то должно найтись $j \geqslant l$ такое, что $\operatorname{ord}_{x}\left(H_{j+1}\right) \leqslant \operatorname{ord}_{x}\left(H_{j}\right)$. Опять будем считать $j$ минимальным числом, для которого выполнены последние два неравенства.

Обозначим $\operatorname{ord}_{x}\left(H_{j}\right)$ через $n$. В силу нашего выбора индексов $l$ и $j$, порядки $H_{j-1}$, $H_{j}$ и $H_{j+1}$ не превосходят $n$, и, дифференцируя формулу (8) по $u_{n+1}$ при $i=j$, получим

$$
\left(D_{y}+F_{u_{x}}\right)\left(\frac{\left(H_{j}\right)_{u_{n}}}{H_{j}}\right)=0,
$$

откуда следует

$$
F_{u_{x}}=D_{y}\left(\ln H_{j}-\ln \left(H_{j}\right)_{u_{n}}\right) .
$$

Сопоставляя последнее соотношение с $F_{u_{x}}=D_{y}(s), \operatorname{ord}_{x}(s)=m<n$, и учитывая, что $H_{j}$ зависит от $u_{n}$ линейно (это видно из формулы (8) и того, что $\operatorname{ord}_{x}\left(H_{i}\right)$ растет при $i=\overline{l-1, j})$, получаем, что уравнение (1) имеет нетривиальный $x$-интеграл $\left(\ln H_{j}-\right.$ $\left.\ln \left(H_{j}\right)_{u_{n}}-s\right)$ порядка $n$. Следовательно, в силу утверждения $1 H_{i}=0$ при некотором $i<n$, а значит, $j<n-1$.

$\mathrm{C}$ другой стороны, $\operatorname{ord}_{x}\left(H_{j}\right)$ не может превосходить $j+1$, т.е. $j \geqslant n-1$, что противоречит полученной выше оценке $j<n-1$. Это противоречие доказывает утверждение.

\section{4. ДОКАЗАТЕЛЬСТВО ОГРАНИЧЕННОСТИ ПОРЯДКОВ ИНВАРИАНТОВ ЛАПЛАСА}

Для доказательства 1-го вспомогательного утверждения, т.е. ограниченности порядков инвариантов Лапласа, нам понадобится соотношение (13), а вернее, его линеаризация.

Напомним, что оператором линеаризации функции $q$ называется дифференциальный оператор

$$
q_{*}=\frac{\partial q}{\partial u}+\sum_{i=1}^{\infty}\left(\frac{\partial q}{\partial v_{i}} D_{y}^{i}+\frac{\partial q}{\partial u_{i}} D_{x}^{i}\right)
$$

Обозначим через $[Z]$ множество операторов вида

$$
\sum_{i=1}^{n} \sum_{j=1}^{n} \zeta_{i j} D_{x}^{i} \circ D_{y}^{j} \circ Z
$$

где $\zeta_{i j}$ - некоторые функции, $n$ - произвольное неотрицательное целое число, а $Z$ - оператор вида (6). Непосредственным вычислением можно убедиться в том, что верны формулы

$$
\begin{aligned}
& \left(D_{x}(q)\right)_{*}=D_{x} \circ q_{*}(\bmod [L]), \\
& \left(D_{y}(q)\right)_{*}=D_{y} \circ q_{*}(\bmod [L]),
\end{aligned}
$$

где через $L$ обозначен оператор линеаризации уравнения (1), заданньй формулой (12). 
Линеаризуя соотношение (13) и учитывая формулы (14), получим

$$
M \circ g_{*}=P \circ L
$$

где $P$ - дифференциальный оператор вида

$$
P=\sum_{i=0}^{k} \xi_{i} D_{x}^{i}+\sum_{i=1}^{m} \xi_{-i} D_{y}^{i}
$$

$\xi_{i}-$ функции, $\xi_{k} \neq 0$, а $M$ задается формулой

$$
M=D_{x} D_{y}+\alpha(x, y) D_{x}+\beta(x, y) D_{y}+\gamma(x, y)
$$

Формальным сопряжением из формулы (15) получаем

$$
L^{\top} \circ P^{\top}=0 \quad\left(\bmod \left[M^{\top}\right]\right)
$$

Напомним, что формальное сопряжение есть линейная операция на множестве дифференциальных операторов, определяемая правилами $D_{x}^{\top}=-D_{x}, D_{y}^{\top}=-D_{y}$ и $(P$ 。 $R)^{\top}=R^{\top} \circ P^{\top}$ для любой пары операторов $P$ и $R$, где $S^{\top}$ обозначает оператор, сопряженный оператору $S$.

Рассмотрим ситуацию, когда ядро оператора вида (17) переводится оператором вида (16) в ядро произвольного оператора (6). Нам потребуются две леммы. Сначала мы их сформулируем, затем покажем, как из них вытекает доказательство интересующего нас утверждения, и лишь затем докажем сами леммы.

Лемма 1. Пусть для некоторого оператора $Z$ вида (6) найдутся оператор $P$ вида (16) $c \xi_{k} \neq 0 u k>0$, а также оператор $M$ вида (17) такие, ито

$$
Z \circ P=0 \quad(\bmod [M])
$$

Тогда существуют оператор

$$
\widehat{P}=\sum_{i=0}^{k+1} \hat{\xi}_{i} D_{x}^{i}+\sum_{i=1}^{m} \hat{\xi}_{-i} D_{y}^{i}, \quad \hat{\xi}_{k+1}=\xi_{k}, \quad \operatorname{ord}_{x}\left(\hat{\xi}_{i}\right) \leqslant \max _{-m \leqslant i \leqslant k} \operatorname{ord}_{x}\left(\xi_{i}\right),
$$

и оператор $\widehat{M}$ вида (17) такие, что

$$
Z \circ \widehat{P}=0 \quad(\bmod [\widehat{M}]) .
$$


Лемма 2. Пусть для некоторого оператора $Z$ вида (6) найдутся оператор $P$ вида (16) $с \xi_{k} \neq 0$ и $k>1$, а также оператор $M$ вида (17) такие, что выполнено соотношение (19). Обозначим $\max _{-m \leqslant i \leqslant k} \operatorname{ord}_{x}\left(\xi_{i}\right)$ через $l$, а главный у-инвариант Лапласа оператора $Z$ - через $K_{0}$. Тогда:

а) выполнено условие $\operatorname{ord}_{x}\left(K_{0}\right) \leqslant \max \{1, l\}$;

б) для у-преобразования Лапласа $Z_{-1}$ оператора $Z$ найдутся оператор

$$
\widetilde{P}=\sum_{i=0}^{k} \tilde{\xi}_{i} D_{x}^{i}+\sum_{i=1}^{m+1} \tilde{\xi}_{-i} D_{y}^{i}, \quad \tilde{\xi}_{k}=K_{0} \xi_{k}, \quad \operatorname{ord}_{x}\left(\tilde{\xi}_{i}\right) \leqslant \max \{1, l\},
$$

и оператор $\widetilde{M}$ вида (17) такие, что

$$
Z_{-1} \circ \widetilde{P}=0 \quad(\bmod [\widetilde{M}]) .
$$

Леммы 1 и 2 в совокупности означают, что верно следуюшее

УТВЕРЖДЕНИЕ 2. Пусть выполнены условия леммы 1. Тогда у-инварианты Лапласа $K_{i}$ оператора $Z$ удовлетворяют условию $\operatorname{ord}_{x}\left(K_{i}\right) \leqslant \max \{1, l\}$, əде $l=$ $\max _{-m \leqslant i \leqslant k} \operatorname{ord}_{x}\left(\xi_{i}\right)$.

Действительно, без нарушения общности можно считать $k>1$, так как, применив лемму 1 , мы можем повысить порядок оператора Р относительно $D_{x}$. Тогда, многократно применяя утверждение “б” леммы 2, а затем применив утверждение “а" леммы 2 к оператору $Z_{-i}$, получим, что порядок главного $y$-инварианта Лапласа оператора $Z_{-i}$ (который, как видно из рассуждений, предшествующих определению 1 , совпадает с $i$-м $y$-инвариантом $K_{i}$ оператора $Z$ ) не превосходит $\max \{1, l\}$, где $l$ - максимальньй из порядков коэффициентов оператора $P$.

Возврашаясь к формуле (18), заметим, что при формальном сопряжении оператора вида (6) его главные $x$ - и $y$-инварианты меняются местами, а следовательно (см. формулы (8), (9)), $y$-инварианты Лапласа оператора $L^{\top}$ совпадают с $x$-инвариантами $H_{i}$ уравнения (1). Тогда в силу утверждения 2 вьполнено $\operatorname{ord}_{x}\left(H_{i}\right) \leqslant \max \{1, \tilde{l}\}$, где $\tilde{l}-$ максимальный из порядков коэффициентов оператора $P^{\top}$ в формуле (18). Таким образом, доказательство ограниченности порядков инвариантов Лапласа линеаризуемого уравнения (1) сводится к доказательству лемм 1 и 2.

ДоКАЗАТЕЛЬСТво ЛЕммы 1 . Обозначим через $\mathcal{K}$ главный $y$-инвариант Лапласа оператора $M$, а через $\widehat{M}$ - оператор

$$
\widehat{M}=\left(D_{y}+\alpha\right) \circ\left(D_{x}+\beta_{1}\right)-\mathcal{K},
$$

где $\beta_{1}=\beta+D_{x}(\ln \mathcal{K})$, если $\mathcal{K} \neq 0$, и $\beta_{1}=\beta$, если $\mathcal{K}=0$. Принимая во внимание, что оператор $M$ можно записать в виде $\left(D_{x}+\beta\right) \circ\left(D_{y}+\alpha\right)-\mathcal{K}$, нетрудно проверить, что верна формула

$$
M \circ\left(D_{x}+\beta_{1}\right)=\left(D_{x}+\beta\right) \circ \widehat{M} .
$$

Воспользовавшись формулами (20) и (19), получим

$$
Z \circ P \circ\left(D_{x}+\beta_{1}\right)=0 \quad(\bmod [\widehat{M}]) .
$$


Ясно, что в качестве искомого оператора $\widehat{P}$ можно взять оператор $P \circ\left(D_{x}+\beta_{1}\right)$, нужно лишь исключить в последнем операторе слагаемые с $D_{y}^{i}$ 。 $D_{x}$, воспользовавшись для этого формулой

$$
D_{x} \circ D_{y}^{i}=\zeta_{-1}^{i}(x, y) D_{x}+\sum_{j=0}^{i} \zeta_{j}^{i}(x, y) D_{y}^{j} \quad(\bmod [M]),
$$

которую нетрудно получить по индукции. Коэффициент при $D_{x}^{k+1}$ в построенном таким образом операторе $\widehat{P}$ будет совпадать с $\xi_{k}$, а порядки прочих коэффициентов оператора $\widehat{P}$ не превзойдут максимального из порядков коэффишиентов оператора $P$, так как $\beta_{1}$ и $\zeta_{j}^{i}$ зависят только от $x$ и $y$. Лемма 1 доказана.

ДОКАЗАТЕЛЬСТво ЛЕМмЫ 2. Индукцией по $i$ нетрудно проверить, что

$$
D_{y} \circ D_{x}^{i}=\eta_{-1}^{i}(x, y) D_{y}+\sum_{j=0}^{i} \eta_{j}^{i}(x, y) D_{x}^{j} \quad(\bmod [M]),
$$

причем при $i>1$ верны формулы

$$
\eta_{i}^{i}=-\alpha, \quad \eta_{i-1}^{i}=\alpha \beta-\gamma-(i-1) \alpha_{x} .
$$

Воспользовавшись формулами (21), (22), исключим из левой части соотношения (19) члены вида $D_{x} \circ D_{y}^{i}$ и $D_{y} \circ D_{x}^{i}$. Полученное в результате этой операции выражение должно уже просто равняться нулю (а не с точностью до $[M]$ ), так как оно не содержит $D_{x}^{i} \circ D_{y}^{i}$ с $i, j \neq 0$. Собирая в этом выражении члены с $D_{x}^{k+1}$ и $D_{x}^{k}$ и учитывая (23), получим

$$
\begin{gathered}
\left(D_{y}+a-\alpha\right)\left(\xi_{k}\right)=0, \\
\left(\gamma-\alpha \beta+(k-1) \alpha_{x}+K_{0}\right) \xi_{k}-\left(D_{y}+a-\alpha\right)\left(\xi_{k-1}\right)=0 .
\end{gathered}
$$

Формула (25) с учетом (24) может быть записана в виде

$$
K_{0}=D_{y}\left(\frac{\xi_{k-1}}{\xi_{k}}\right)+\alpha \beta-\gamma-(k-1) \alpha_{x} .
$$

Последнее соотношение доказывает утверждение "а" леммы 2.

Применяя формулу (7) к соотношению (19), получим

$$
\left(D_{y}+a_{1}\right) \circ Z \circ P=Z_{-1} \circ\left(D_{y}+a\right) \circ P=0 \quad(\bmod [M]) .
$$

Нетрудно видеть, что

$$
\begin{aligned}
\left(D_{y}+a\right) \circ P= & \sum_{i=0}^{k}\left(\left(D_{y}+a\right)\left(\xi_{i}\right) D_{x}^{i}+\xi_{i} D_{y} \circ D_{x}^{i}\right)+ \\
& +\sum_{i=1}^{m}\left(\left(D_{y}+a\right)\left(\xi_{-i}\right) D_{y}^{i}+\xi_{-i} D_{y}^{i+1}\right) .
\end{aligned}
$$


В этом соотношении исключим все члены с $D_{y} \circ D_{x}^{i}$, используя формулу (22). Ясно, что порядки $\operatorname{ord}_{x}\left(\tilde{\xi}_{i}\right)$ коэффициентов полученного в результате этого действия оператора

$$
\widetilde{P}=\sum_{i=0}^{k} \tilde{\xi}_{i} D_{x}^{i}+\sum_{i=1}^{m+1} \tilde{\xi}_{-i} D_{y}^{i}
$$

могут возрасти либо из-за дифференцирования $D_{y}$ (это может произойти лишш в случае $\operatorname{ord}_{x}\left(\xi_{i}\right)=0$, и тогда $\left.\operatorname{ord}_{x}\left(D_{y}\left(\xi_{i}\right)\right) \leqslant 1\right)$, либо за счет того, что $\operatorname{ord}_{x}(a)>l$. Но в силу $(24) \operatorname{ord}_{x}(a) \leqslant \max \left\{1, \operatorname{ord}_{x}\left(\xi_{k}\right)\right\}$. Таким образом, $\operatorname{ord}_{x}\left(\tilde{\xi}_{i}\right) \leqslant \max \{1, l\}$.

Более тшательные вычисления с применением формулы (23) показывают, что

$$
\begin{gathered}
\tilde{\xi}_{k}=\left(D_{y}+a-\alpha\right)\left(\xi_{k}\right) \\
\tilde{\xi}_{k-1}=\left(D_{y}+a-\alpha\right)\left(\xi_{k-1}\right)-\left(\gamma-\alpha \beta+(k-1) \alpha_{x}\right) \xi_{k} .
\end{gathered}
$$

Учитывая (24), (25), из последних формул получаем $\tilde{\xi}_{k}=0, \tilde{\xi}_{k-1}=K_{0} \xi_{k}$. Кроме того, так как по построению $\widetilde{P}=\left(D_{y}+a\right) \circ P(\bmod [M])$, из формулы $(26)$ следует

$$
Z_{-1} \circ \widetilde{P}=0 \quad(\bmod [M])
$$

Применение леммы 1 к последней формуле завершает доказательство утверждения "б” леммы 2. Лемма 2 доказана.

Благодарности. Автор благодарен В.В. Соколову и А.В. Жиберу за внимание к работе и полезные замечания.

Работа выполнена при финансовой поддержке Российского фонда фундаментальных исследований (грант № 99-01-00431).

\section{Список литературы}

[1] I. M. Anderson, N. Kamran. The variational bicomplex for second order scalar partial differential equations in the plane. Preprint. Montreal: Centre de Recherches Mathematiques, 1994.

[2] А. В. Жибер, В. В. Соколов, С. Я. Стариев. Доклады РАН. 1995. Т. 343. С. 746.

[3] V. V. Sokolov, A. V. Zhiber. Phys. Lett. A. 1995. V. 208. P. 303.

[4] I. M. Anderson, M. Juras. Generalized Laplace invariant and the Method of Darboux. Preprint. Montreal: Centre de Recherches Mathematiques, 1995.

[5] А.В. Жибер, В.В. Сожолов. Преобразования Лапласа в классификации интегрируемых квазилинейных уравнений. В сб.: Проблемы механики и управления. Т. 2. Ред. Р. Р. Мавлютов. Уфа: Институт механики РАН, 1996. С. 112.

[6] С.Я. Старцев. Об инвариантах Лапласа систем гиперболических уравнений. В сб.: Комплексный анализ, дифференциальные уравнения, численные методы и приложения. Т. 3. Ред. И.Ф. Красичков-Терновский, Л.А. Калякин, М.Д. Рамазанов. Уфа: Институт математики с ВЦ УНЦ РАН, 1996. С. 144.

[7] С. Я. Старцев. ТМФ. 1998. Т. 116. № 3. С. 336.

[8] С. Я. Старцев. Аналог вариационной производной для гиперболических уравнений. В сб.: Проблемы математики и теории управления. Уфа: УГАТУ, 1998. С. 174

[9] V. V. Sokolov, S. I. Svinolupov. Eur. J. Appl. Math. 1995. V. 6. P. 145.

[10] E. Goursat. Bull. Soc. Math. France. 1897. V. 25. P. 36.

Поступила в редакцию 25.I.1999 г. 